\section{Aortitis caused by Abiotrophia defectiva: Description of two cases}

\author{
David Nygren, ${ }^{1}$ Martin Älverbrandt, ${ }^{2}$ \\ Torgny Sunnerhagen, ${ }^{1}$ Erika Fagman, ${ }^{3}$ \\ Ellen Ostenfeld, ${ }^{1}$ Magnus Rasmussen ${ }^{1}$ \\ ${ }^{1}$ Lund University and Skåne University \\ Hospital, Lund; ${ }^{2}$ Borås Hospital; \\ ${ }^{3}$ University of Gothenburg and \\ Sahlgrenska University Hospital, \\ Gothenburg, Sweden
}

\begin{abstract}
Abiotrophia defectiva is a well-known endocarditis pathogen, however it has never been described as a cause of primary aortitis. Here we describe the first published case of thoracic aortitis and an unusual case of aortic graft infection due to A. defectiva, which were both managed conservatively.
\end{abstract}

\section{Introduction}

Abiotrophia defectiva is a commensal of the human oral, gastrointestinal and genitourinary tract, ${ }^{1}$ and part of the fastidious nutritionally variant streptococci (NVS)..$^{2,3}$ NVS infections carry a high number of bacteriological failures and relapses. ${ }^{4}$ Through antimicrobial sensitivity testing (AST), Alberti et al. demonstrated that only $11 \%$ of $A$. defectiva isolates were sensitive to penicillin, whereas $70 \%$ were intermediate and $19 \%$ resistant. ${ }^{5}$ Further studies verified this, and showed that 92,98 and $100 \%$ respectively were sensitive to ceftriaxone..$^{5-7}$

Abiotrophia is primarily known as a causative agent of infective endocarditis, $3,4,8$ but vascular infections due to $A$. defectiva have also been described co-occurring with endocarditis or endovascular grafts., 910 To our knowledge this bacterium has not been described as a cause of primary aortitis yet. Here, we describe two cases of aortitis caused by $A$. defectiva with different pathogenesis.

\section{Case Report \#1}

A 68-year-old female presented in March 2017 with fever, intermittent chest pain and dyspnea for more than a month. Her medical history included type 2 diabetes mellitus, smoking, hypertension and chronic renal failure grade III.

Her vital parameters were normal and her temperature was $37.8^{\circ} \mathrm{C}$. Physical examination was normal except for a known precordial systolic murmur. White blood cell count (WBC) was $16 \times 10^{9} / \mathrm{L}$ and CRP $255 \mathrm{mg} / \mathrm{L}$. Troponin T and electrocardiography (ECG) were normal. A computed tomography (CT) of the thorax showed pericardial fluid and $2 \mathrm{~cm}$ of bilateral pleural effusion. Transthoracic echocardiography demonstrated a $0.5-1 \mathrm{~cm}$ pericardial effusion, a mild aortic stenosis but no signs of endocarditis. Two sets of blood cultures were drawn and empirical treatment with cefotaxime was started.

In $1 / 4$ blood culture bottles (BACTEC Plus Aerobic and Lytic Anaerobic ( $) A$. defectiva was identified using MALDI-TOF MS. Further evaluation with a transesophageal echocardiography (TEE) revealed two separate vegetations located to the aortic wall in the aortic arch and suspected signs of aortitis (Figure 1A-C) but no signs of endocarditis. ECG-gated CTangiography confirmed signs of aortitis and two separate vegetations in the aortic arch (Figure 1D-F). To narrow therapy, according to MICs in AST derived from E-tests (Table 1), treatment was changed to penicillin $G$ plus rifampicin. This led to the return of fever and worsening symptoms, which is why penicillin $G$ was changed for ampicillin to maintain a narrow spectrum therapy, despite a slightly higher MIC in Etest (Table 1, E-test). However, the fever persisted why cefotaxime combined with rifampicin and ciprofloxacin was initiated with a continuous improvement from then on. The pericardial effusion decreased and the pleural fluid was drained bilaterally, with negative cultures and 16S RNA PCR. After seven weeks of intravenous antibiotics she was discharged, with an individualized and prolonged treatment with four months of oral ciprofloxacin and rifampicin. Six months after cessation of therapy the patient was well, afebrile and with normalized CRP.

\section{Case Report \#2}

A 47-year-old male presented in March 2017 due to intermittent and increasing chest pain for a week. His medical history included surgery of a bicuspid stenotic aortic valve and an aneurysm of the ascending aorta with a bovine biological aortic valve and a supracoronary Polythese $\AA$ graft two months prior. His vital parameters were normal except for a temperature of $39^{\circ} \mathrm{C}$. Physical examination demonstrated a systolic murmur over the aortic valve. WBC was $13 \times 10^{9} / \mathrm{L}$ and CRP $34 \mathrm{mg} / \mathrm{L}$. Troponin $\mathrm{T}$ was normal and ECG was unchanged. Two sets of blood cultures were drawn and empirical treatment with isoxazolylpenicillin was initiated. A CT of the thorax
Correspondence: David Nygren, Hälsogatan 3, Skåne University Hospital Lund, Lund SE221 85, Sweden.

Phone: +46.46.171192 - Fax: +46.46.76002. E-mail: david.nygren@med.lu.se

Key words: Abiotrophia, Aortitis, Prosthesis Related Infections, Aortic Graft Infection.

Contributions: DN wrote the manuscript except for Case 2, which was written by MÄ. TS performed the antimicrobial sensitivity testing described in the paper. EF and EO contributed with acquisition and interpretation of the figures described in the paper. MR coordinated the work and provided the design for this case report.

Conflict of interest: the authors declare no conflict of interest.

Funding: this work was supported by the Swedish Government Fund for Clinical Research (ALF).

Received for publication: 14 May 2018.

Revision received: 15 June 2018.

Accepted for publication: 16 June 2018.

This work is licensed under a Creative Commons Attribution-NonCommercial 4.0 International License (CC BY-NC 4.0).

CCopyright D. Nygren et al., 2018

Licensee PAGEPress, Italy

Infectious Disease Reports 2018; 10:7746

doi:10.4081/idr.2018.7746

showed a ring of soft tissue attenuation around the graft (Figure 2A), indicative of inflammation that could be a normal postoperative finding, but in which it was hard to exclude infection. TEE showed no signs of endocarditis.

In 4/4 blood culture bottles (BacT/ALERT FA/FN Plus ${ }^{\circledR}$ ) A. defectiva was identified using MALDI-TOF MS and treatment for a possible prosthetic valve endocarditis was initiated with penicillin $G$ and tobramycin. Initial improvement was seen, however, three weeks after the initiation of therapy an itchy rash and a fever developed. A beta-lactam reaction was suspected and penicillin $\mathrm{G}$ was replaced with cefotaxime, but fever persevered. TEE then demonstrated an enlargement of the soft tissue adjacent to the aortic graft, for which reason an aortic graft infection (AGI) was suspected; cefotaxime and tobramycin were changed to meropenem and vancomycin and the patient was transferred for preoperative assessment at the regional Department for Thoracic Surgery and performed an ECG-gated CT-angiography which confirmed progress of the inflammation in the 
soft tissue surrounding the graft (Figure 2B). After the antibiotic switch, the patient stabilized, CRP decreased, the fever and the rash disappeared. A multidisciplinary discussion and a risk-benefit assessment resulted in the decision not to operate him, due to the risk of severe complications and the recently stabilized situation. Intravenous vancomycin was given for an additional 3.5 weeks, after which the patient was asymptomatic and CRP almost normalized, why treatment with clindamycin and rifampicin was initiated. However, after two months, an alanine transaminase (ALT) elevation occurred. Since extended AST had shown high MIC for clindamycin in solution (Table 1), the treatment was changed to amoxicillin and ALT normalized. Nine months into treatment, a ${ }^{18} \mathrm{~F}$-FDG Positron Emission Tomography-CT was performed which showed an increased ${ }^{18} \mathrm{~F}-\mathrm{FDG}$ uptake around the aortic graft and the surrounding soft tissue, indicating a chronic AGI (Figure 2C), why a life-long suppressive therapy was planned. After 10 months follow up, the patient was well and CRP normalized.

\section{Discussion}

For the first time in the literature we describe an isolated aortic infection due to A. defectiva, as well as an AGI, two cases with markedly different pathogenesis. Both cases were treated conservatively. Guidelines, however based on weak evidence, are available for endocarditis caused by Abiotrophia, ${ }^{11,12}$ but are lacking for the treatment of aortitis, ${ }^{13}$ why individualized treatments were chosen according to clinical response and AST.

According to Sörelius et al. mycotic abdominal aortic aneurysm are defined as a combination of three criteria, i.e. clinical presentation suggestive of infection, laboratory markers suggestive of infection and radiological findings on $\mathrm{CT}$ or MRI. ${ }^{14} \mathrm{In}$ case number one, however, a case with a thoracic location identified prior to the development of an aneurysm, all of these three criteria were met. A. defectiva was identified in only one blood culture using MALDI-TOF MS, however this known endocarditis pathogen, $3,4,8-10$ was regarded as a significant finding due to its proposed endovascular adhesiveness and the clinical and radiological evidence of infectious aortitis. $^{15}$

A proposed definition, currently under

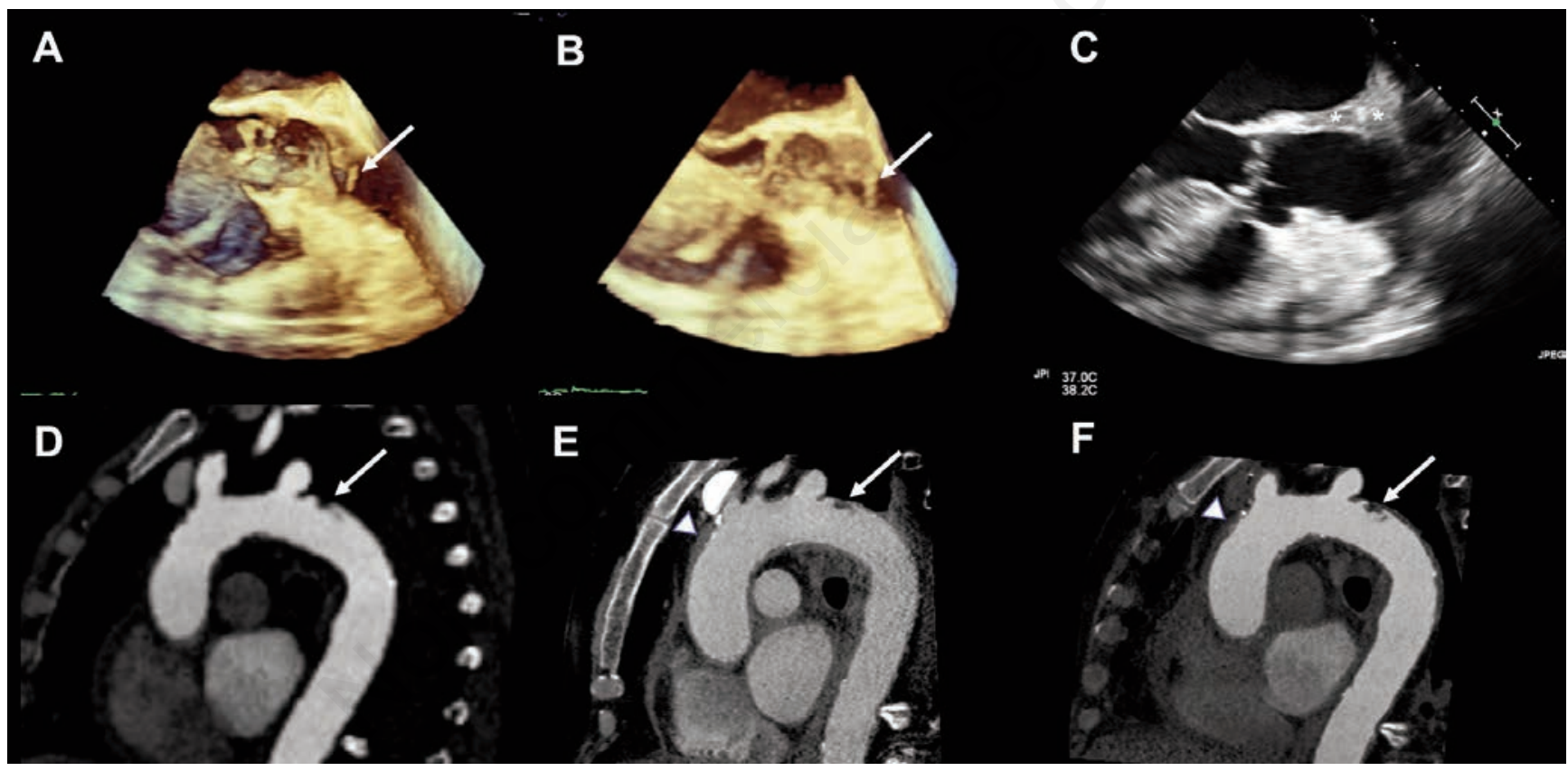

Figure 1. TEE (A-C) of the ascending aorta and aortic valve, and ECG-gated iodine-contrast enhanced CT angiography of the thoracic aorta (D-F) from three different time points. A) Three-dimensional TEE at initial examination. Two mobile structures were seen; one in the ascending aorta and one in the aortic arch. The white arrow indicates the $8 \mathrm{~mm}$ mobile structure in the ascending aorta at an atherosclerotic plaque approximately $3 \mathrm{~cm}$ above the aortic valve suspected to be a vegetation. B) Three-dimensional TEE a week after treatment. White arrow shows unchanged size of the mobile structure in the ascending aorta. C) Two-dimensional TEE a month after initiated treatment. The mobile structure in the ascending aorta has regressed and is not visible, but the aortic wall was thickened $(*)$ which indicated a possible infective abscess. At examination 2 months after initiated treatment the vegetation and the thickened wall in the ascending aorta had fully regressed, but the mobile structure in the aortic arch was unchanged. D) CT angiography of the aorta at initial examination. Several partly calcified plaques were seen in the thoracic aorta. About $3 \mathrm{~cm}$ above the aortic valve a low attenuating $2 \times 2 \mathrm{~mm}$ large structure protruding into the aortic lumen was seen. The aortic wall was thickened in the ascending aorta and the aortic arch. In the aortic arch just distal of the left subclavian artery a partly calcified plaque with a low attenuating mobile structure of about $3 \mathrm{~mm}$ attached was seen (white arrow). No abscess was present. E) CT angiography of the aorta one week after initiated treatment. The mobile structures in the ascending aorta and the aortic arch (white arrow) were unchanged. The thickening of the aortic wall (white arrowhead) persisted, however with some progress in the aortic arch wall, suggesting progress in the infective aortitis. No abscess was present. F) CT angiography of the aorta one month after initiated treatment. The mobile structure in the ascending aorta was hardly identifiable and the structures in the aortic arch (white arrow) were unchanged. The thickening of the aortic wall (white arrowhead) and the aortic arch wall had progressed even further with increased thickening of the aortic wall at the aortic root dorsally of the left atrium. No abscess was present. At examination one and a half months after initiated treatment the aortic wall thickening had regressed and the mobile structure in the ascending aorta was not visualized. 
validation, defines AGI by clinical/pathological, radiological and laboratory criteria, where one major and one minor criteria are considered an evident diagnosis of AGI, while three minor criteria are considered a suspected AGI ${ }^{16}$ In regard to this definition, case number two exhibited no major, but four minor criteria, i.e. fever with AGI the most likely cause, radiological signs of perigraft inflammation, positive blood cultures and elevated inflammatory markers pointing towards a suspected AGI. The short time between the operation and the infection suggests that the bacteria were already introduced at surgery.

In case one, relapse occurred after the change of cefotaxime to penicillin $\mathrm{G}$, despite the low MIC-values on E-test. However, a high minimum bactericidal con- centration $(\mathrm{MBC})$ for penicillin was found on extended AST (Table 1). Nevertheless, the same MIC/MBC pattern was seen for cefotaxime, which when used, led to clinical improvement. This discrepancy between in vitro sensitivity and effect of antibiotics in vivo for NVS infections has been known for some time, ${ }^{4}$ and could be in part explained by their in vivo production of exopolysaccharides, i.e. biofilm. ${ }^{17} \mathrm{MIC}$-values of clindamycin determined through serial dilution were strikingly increased compared to the E-test for both isolates, a pattern that persisted through several repetitions of testing (Table 1). This discrepancy remains without an explanation, and in addition to a potential adverse reaction with elevated ALT in patient two, this led to an unwillingness to use clindamycin and thus to a change of therapy. Finally, rifampicin had low MIC- and MBC-values in both isolates, which is why it was used in both patients due to its known effects on the aforementioned biofilm. ${ }^{18}$

\section{Conclusions}

This report describes for the first time in the literature a case of infectious aortitis caused by $A$. defectiva, as well as an unusual case of aortic vascular graft infection caused by the same pathogen, where in both cases remission was seen with conservative treatment. Therapeutic challenges in the treatment of $A$. defectiva are illustrated and examined in this paper.

Table 1. Antibiotic susceptibility of the two isolates. Concentrations shown as $\mu \mathrm{g} / \mathrm{ml}$. Minimal inhibitory concentration (MIC), was measured by E-test and serial dilution in nutrient broth. ${ }^{19} \mathrm{MBC}$ (minimum bactericidal concentration, the lowest antibiotic concentration to cause a reduction in colony forming units by $\mathbf{9 9 . 9 \%}$ in 24 hours) was also tested in nutrient broth, with bacteria then plated on agar plates to measure the number of surviving cells. MIC in serial dilution and MBC was not tested (NT) for vancomycin and gentamicin.

\begin{tabular}{|c|c|c|c|c|c|c|}
\hline \multirow[t]{2}{*}{ Antibiotic } & \multicolumn{2}{|c|}{ MIC (E-test) } & \multicolumn{2}{|c|}{ MIC (serial dilution) } & \multicolumn{2}{|c|}{ MBC } \\
\hline & Case 1 & Case 2 & Case 1 & Case 2 & Case 1 & Case 2 \\
\hline Penicillin G & 0.13 & 0.25 & 0.06 & 0.13 & 1 & 1 \\
\hline Cefotaxime & 0.25 & 0.5 & 0.25 & 0.5 & 1 & 1 \\
\hline Ampicillin & 0.5 & 0.25 & 0.03 & 0.06 & 0.5 & 0.13 \\
\hline Vancomycin & 1 & 2 & NT & NT & NT & NT \\
\hline Gentamicin & 4 & 4 & NT & NT & NT & NT \\
\hline Ciprofloxacin & 0.25 & 0.25 & 0.5 & 1 & 4 & 4 \\
\hline Rifampicin & $<0.002$ & $<0.002$ & 0.0005 & 0.001 & 0.06 & 0.004 \\
\hline Clindamycin & 0.25 & 0.25 & 128 & 32 & $>128$ & 128 \\
\hline
\end{tabular}

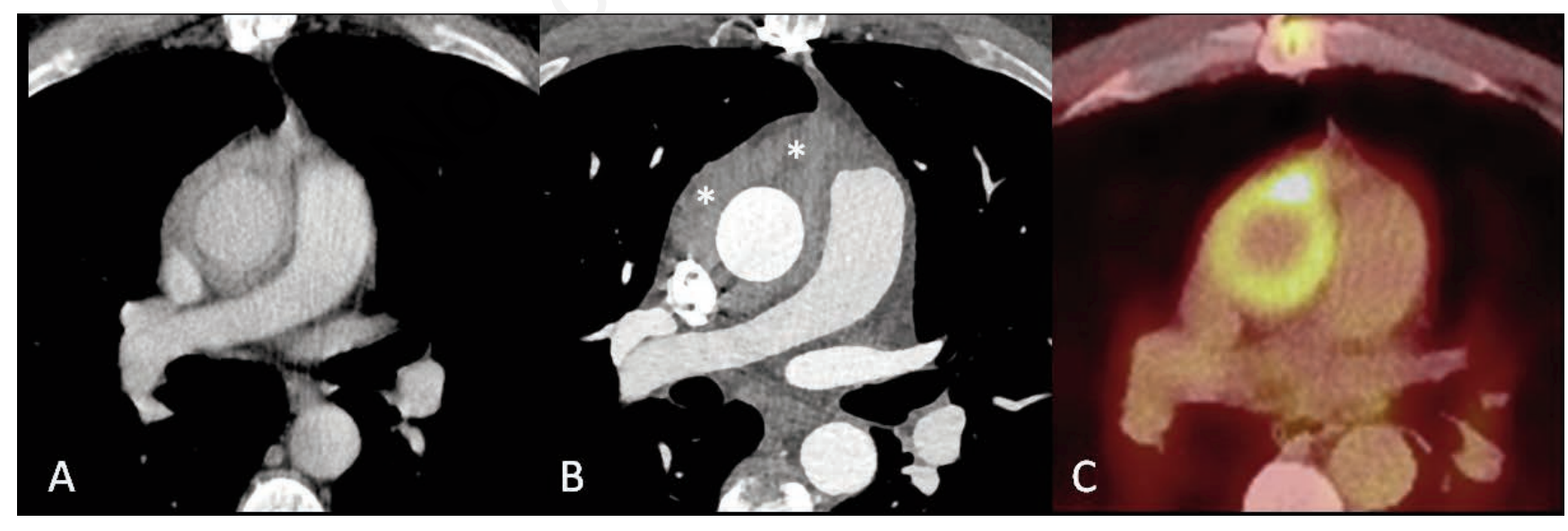

Figure 2. Transaxial images of the ascending aortic graft from three different time points. A) Contrast enhanced CT performed two months postoperatively shows a ring of soft tissue attenuation around the graft. In this early postoperative phase, it is difficult to distinguish postoperative inflammation from infection. B) ECG-gated contrast enhanced CT three months postoperatively shows clear progression of areas of soft tissue attenuation around the graft $*^{*}$ indicating infection. C) 18F-FDG PET/CT performed eleven months after the first CT scan shows increased 18F-FDG uptake circumferentially around the graft with a focal spot of high uptake ventrally in a region with soft tissue attenuation Increased 18F-FDG uptake in an early postoperative phase after aortic graft implantation or valve replacement can be due to normal postoperative inflammation and should be interpreted cautiously in the search for an infective process. However, in the present case 18F-FDG PET/CT was performed eleven months after surgery and the findings indicate persisting infection. 


\section{References}

1. Ruoff KL. Nutritionally variant streptococci. Clin Microbiol Rev 1991;4:18490.

2. Kawamura Y, Hou XG, Sultana F, et al. Transfer of Streptococcus adjacens and Streptococcus defectivus to Abiotrophia gen. nov. as Abiotrophia adiacens comb. nov. and Abiotrophia defectiva comb. nov., respectively. Int J Syst Bacteriol 1995;45:798-803.

3. Bouvet A. Human endocarditis due to nutritionally variant streptococci: Streptococcus adjacens and Streptococcus defectivus. Eur Heart J 1995;16:24-7.

4. Stein DS, Nelson KE. Endocarditis due to nutritionally deficient streptococci: therapeutic dilemma. Rev Infect Dis 1987;9:908-16.

5. Alberti MO, Hindler JA, Humphries RM. Antimicrobial Susceptibilities of Abiotrophia defectiva, Granulicatella adiacens, and Granulicatella elegans. Antimicrob Agents Chemother 2015; 60:1411-20.

6. Mushtaq A, Greenwood-Quaintance $\mathrm{KE}$, Cole NC, et al. Differential Antimicrobial Susceptibilities of Granulicatella adiacens and Abiotrophia defectiva. Antimicrob Agents Chemother 2016;60:5036-9.

7. Prasidthrathsint K, Fisher MA. Antimicrobial Susceptibility Patterns among a Large, Nationwide Cohort of Abiotrophia and Granulicatella Clinical Isolates. J Clin Microbiol 2017; 55:1025-31.

8. Tellez A, Ambrosioni J, Llopis J, et al.
Epidemiology, Clinical Features, and Outcome of Infective Endocarditis due to Abiotrophia Species and Granulicatella Species: Report of 76 Cases, 2000-2015. Clin Infect Dis 2018;66:104-11.

9. Raff GW, Gray BM, Torres A, Jr., Hasselman TE. Aortitis in a child with Abiotrophia defectiva endocarditis. Pediatr Infect Dis J. 2004;23:574-6.

10. Senn L, Entenza JM, Greub G, et al. Bloodstream and endovascular infections due to Abiotrophia defectiva and Granulicatella species. BMC Infect Dis 2006;6.

11. Baddour LM, Wilson WR, Bayer AS, et al. Infective Endocarditis in Adults: Diagnosis, Antimicrobial Therapy, and Management of Complications: A Scientific Statement for Healthcare Professionals From the American Heart Association. Circulation 2015; 132:1435-86.

12. Habib G, Lancellotti P, Antunes MJ, et al. ESC Guidelines for the management of infective endocarditis: The Task Force for the Management of Infective Endocarditis of the European Society of Cardiology (ESC). Endorsed by: European Association for CardioThoracic Surgery (EACTS), the European Association of Nuclear Medicine (EANM). Eur Heart J 2015;36:3075-128.

13. Erbel R, Aboyans V, Boileau C, et al. ESC Guidelines on the diagnosis and treatment of aortic diseases: Document covering acute and chronic aortic diseases of the thoracic and abdominal aorta of the adult. The Task Force for the
Diagnosis and Treatment of Aortic Diseases of the European Society of Cardiology (ESC). Eur Heart J 2014;35:2873-926.

14. Sörelius K, Wanhainen A, Furebring M, et al. Nationwide Study of the Treatment of Mycotic Abdominal Aortic Aneurysms Comparing Open and Endovascular Repair. Circulation 2016;134:1822-32.

15. Senn L, Entenza JM, Prod'hom G. Adherence of Abiotrophia defectiva and Granulicatella species to fibronectin: is there a link with endovascular infections? FEMS Immunol Med Microbiol 2006;48:215-7.

16. Lyons OT, Baguneid M, Barwick TD, et al. Diagnosis of Aortic Graft Infection: A Case Definition by the Management of Aortic Graft Infection Collaboration (MAGIC). Eur J Vasc Endovasc Surg 2016;52:758-63.

17. Kiernan TJ, O'Flaherty N, Gilmore R, et al. Abiotrophia defectiva endocarditis and associated hemophagocytic syndrome - a first case report and review of the literature. Int $J$ Infect Dis 2008;12:478-82.

18. Zimmerli W, Widmer AF, Blatter M, et al. Role of rifampin for treatment of orthopedic implant-related staphylococcal infections: a randomized controlled trial. Foreign-Body Infection (FBI) Study Group. JAMA 1998;279:153741.

19. Jorgensen JH, Ferraro MJ. Antimicrobial susceptibility testing: a review of general principles and contemporary practices. Clin Infect Dis 2009;49:1749-55. 